\title{
Les fondements métaphysiques des cosmo-théosophies africaines: une lecture à partir des textes
}

\section{Os fundamentos metafísicos das cosmo-teosofias africanas: uma leitura à partir dos textos}

The metaphysical founding principles of african cosmotheosophy: a reading from the texts

Mahougnon Venance Sinsin ${ }^{1}$ João dos Santos Barbosa Neto

${ }^{1}$ Doutorado em Filosofia pela Università Pontificia Salesiana, Roma. Mestrado em Filosofia pela Université d'Abomey-Calavi, Benin e Mestrado em Filosofia pela Università Pontificia Salesiana, Roma. Bacharel em Linguística e Ciências da Comunicação pela Université Nationale du Bénin, em Filosofia pelo Institut de Philosophie et Sciences Humaines, Togo, e em Teologia pelo Instituto Teologico San Tommaso, Italia. Salesiano presbítero. Professor de Lógica e de Epistemologia na Faculdade de Filosofia da Pontifícia Universidade Salesiana em Roma. E-mail: sinsin@unisal.it, Orcid: http://orcid.org/0000-0003-3157-2880

${ }^{2}$ Doutorando em Teologia Pastoral pela UPS/ITÁLIA. Mestre em Teologia Pastoral pela UPS/ITÁLIA. Pós-graduado latu senso em Counseling pelo IATES/ PR, e em Psicopedagogia pela Universidade Católica Dom Bosco (UCDB/Portal Educação). Licenciado em Filosofia pela UCDB e bacharel em Teologia pela UPS/ITÁLIA. Salesiano presbítero. E-mail: joaoneto@missaosalesiana.org.br, Orcid: http://orcid.org/0000-0003-1592-1737 
Résumé: Cette étude examine les fondements métaphysiques de la pensée africaine classique et traditionnelle. Elle s'appuie en particulier sur une herméneutique de textes "cosmo-théosophiques» égyptiens, dogon, fang, ciluba. Les questions traitées à partir de l'étude des textes sont les suivantes: l'ontogenèse ou l'origine des choses, l'existence, l'essence et le mouvement, l'union et la parenté des éléments, la diversité et la distinction des étants. II ressort de l'analyse que les cosmo-théosophies africaines se fondent sur une métaphysique qu'on pourrait qualifier de «réalisme significationnel».

Keywords: cosmo-théosophie; metaphysique; hermeneutique; Afrique.

Resumo: Este estudo examina os fundamentos metafísicos do pensamento africano clássico e tradicional. Ele se baseia em particular em uma hermenêutica dos textos "cosmoteosóficos" egípcios, dogonianos, fanguianos, cilubanos. As questões tratadas a partir do estudo dos textos são os seguintes: a ontogenese ou a origem das coisas, a existência, a essência e a tornar, a união e o parentesco dos elementos, a diversidade e a distinção dos seres. O parecer à partir das análises é que las cosmos-teosofias africanas se fundem sobre uma metafísica que pode ser qualificada de «réalisme significationne/».

Palavra-chave: cosmo-teosofia; metafísica, hermenêutica; África.

\begin{abstract}
This study examines the metaphysical foundations of classical and traditional African thought. It is based on a hermeneutics of Egyptian, Dogon, Fang, and Ciluba "Cosmo-Theosophical" texts. The questions dealt with from the study of the texts are as follows: the ontogenesis or origin of things, the existence, the essence and the becoming of things, the union and kinship of elements, the diversity and distinction of beings. The analysis shows that African cosmo-theosophies are based on a metaphysic vision that could be called "realism significationnel".
\end{abstract}

Keywords: cosmo-theosophy; metaphysics; hermeneutics; Africa. 


\section{INTRODUCTION}

A dessein nous éviterons d'utiliser le terme «cosmogonie», non pas en raison de sa signification étymologique (explication de l'origine-génésthai) gon - du kósmos), mais plutôt en raison de l'usage ambigu qui en a été fait dans les études ethnologiques. En ethnologie, en effet, on entend généralement par «cosmogonie» le mythe de la création selon la vision collective d'un peuple. C'est précisément cette idée d'une pensée collective indiscriminée qui nous répugne et qui nous pousse à adopter une autre terminologie. La notion de "cosmogonie" renvoie par ailleurs à la genèse et à la généalogie des dieux (théogonie). Or, cette question n'entre pas dans le cadre de nos préoccupations. Pour toutes ces raisons, nous préférons utiliser le terme «cosmo-théosophie». Par ce vocable nous désignons des formes de spéculation théosophique, élaborées par des Ecoles Initiatiques, et dont la finalité est de proposer des tentatives d'explication de l'origine et de la structure du monde. Par «théosophie», nous entendons précisément une pensée spéculative qui allie la réflexion rationnelle rigoureuse à la réflexion théologique intuitive.

La finalité de l'étude n'est pas de proposer une herméneutique exhaustive des cosmo-théosophies examinées, mais d'explorer les principes métaphysiques qui les fondent, c'est-à-dire les postulats et les présupposés fondamentaux sur lesquels elles reposent. Le corpus de textes que nous aurons à explorer provient de quatre Ecoles Initiatiques: (i) l'Ecole Egyptienne, en particulier celle onto-cosmologique, dont certains des textes sont traduits et commentés par Théophile Obenga (1990); (ii) l'Ecole Dogon, en particulier celle qui se revendique de la filiation initiatique du Vénérable Ogotemmêli; (iii) I’Ecole Fang, en particulier celle qui se réclame de la filiation "mvettique" du Vénérable Zue-Nguema ; (iv) l'Ecole Ciluba, en particulier celle dont les maîtres sont cités par Henri Morlighem dans la Préface de Une Bible noire. Cosmogonie bantu (1973).

La réflexion s'organise autour des questions suivantes: I'ontogenèse ou l'origine des choses (nǔdò), l'existence, l'essence et le mouvement (nǔť̌n, nǔnyí, nǔdannǔ), l'union et la parenté des éléments (nǔdòkpó), la diversité et la distinction des étants (nǔvovo, nŭgbonvo). Les notions mises 
entre parenthèses proviennent de la langue fongbe du Bénin. Puisque nous les utiliserons comme des concepts de base, il convient d'en donner succinctement le sens étymologique:

Tableau 1 - Concepts métaphysiques en langue fongbe (Bénin)

\begin{tabular}{|l|l|l|}
\hline \multicolumn{1}{|c|}{ Termes } & \multicolumn{1}{|c|}{ Décomposition des termes } & \multicolumn{1}{c|}{ Traduction littérale } \\
\hline Nǔdò & «nǔ», chose + «dò», source, abîme & Le fondement de la chose \\
\hline Nǔťn & «nǔ», chose + «tǐn», exister & $\begin{array}{l}\text { L'existence de la chose ou la } \\
\text { chose existante }\end{array}$ \\
\hline Nǔnyí & $\begin{array}{l}\text { «nǔ», chose + «nyí / ny̌̌ être, } \\
\text { nature }\end{array}$ & L'être ou la nature de la chose \\
\hline Nǔvovo & «nǔ», chose + «vovo», divers & La diversité des choses \\
\hline Nǔgbonvo & «nǔ»+ «gbonvo», se différentier & La différentiation des choses \\
\hline Nǔdòkpj́ & $\begin{array}{l}\text { «nǔ»+ «dò», rester, demeurer + } \\
\text { «kpǰ, ensemble }\end{array}$ & L'union des choses \\
\hline Nǔkpíkpé & «nǔ»+ «kpíkpé», complet, parfait & La perfection des choses \\
\hline Nǔdannǔ & $\begin{array}{l}\text { «nǔ»+ «dǎn», mouvoir + «nǔ», } \\
\text { chose }\end{array}$ & La chose qui meut la chose \\
\hline Nyínyí & Réduplication du verbe «nyí», être & $\begin{array}{l}\text { Etre ou advenir } \\
\text { perpétuellement }\end{array}$ \\
\hline Nǔdyj́ & «nǔ»+ «dyว̌», changer, modifier & $\begin{array}{l}\text { Le changement ou la } \\
\text { modification de la chose }\end{array}$ \\
\hline
\end{tabular}

Source: Propre élaboration.

Au terme de l'étude, nous essayerons d'affronter une problématique qui, depuis plus d'une trentaine d'années, suscitent de vives discussions parmi les philosophes africains: quelle vision métaphysique dominante émerge des cosmo-théosophies africaines? Notre hypothèse est que les textes examinés traduisent une métaphysique qu'on pourrait qualifier de «réalisme significationnel». Nous expliciterons le sens de ce concept.

\section{LES COSMO-THÉOSOPHIES ET L'ORDRE DU SAVOIR DANS LES ANCIENNES SOCIÉTÉS AFRICAINES}

On peut distinguer, dans les anciennes sociétés africaines, trois ordres de pensée que l'ethnologie coloniale a souvent confondus. Nous les schématisons comme suit: 
Tableau 2 - La structure du savoir dans les anciennes sociétés africaines

\begin{tabular}{|c|c|c|}
\hline & Organisation des savoirs & $\begin{array}{l}\text { Typologie des } \\
\text { pensées }\end{array}$ \\
\hline $\begin{array}{l}\text { Niveau A } \\
\text { (Supérieur) }\end{array}$ & $\begin{array}{l}\text { Systèmes de connaissances sur } \\
\text { l'univers, les êtres vivants, le Divin, les } \\
\text { nombres et les formes... } \\
\text { (SAVOIRS THEORIQUES) } \\
\downarrow\end{array}$ & $\begin{array}{l}\text { Pensée } \\
\text { spéculative et } \\
\text { scientifique }\end{array}$ \\
\hline $\begin{array}{c}\text { Niveau B } \\
\text { (intermédiaire) }\end{array}$ & $\begin{array}{l}\text { Croyances, codes de vie, sagesses, } \\
\text { pratiques cultuelles, arts et techniques } \\
\text { (SAVOIRS PRATIQUES) }\end{array}$ & $\begin{array}{c}\text { Pensée religieuse, } \\
\text { morale et } \\
\text { pratique }\end{array}$ \\
\hline $\begin{array}{l}\text { Niveau C } \\
\text { (inférieur) }\end{array}$ & $\begin{array}{l}\text { Us et coutumes, légendes, folklore } \\
\text { (SAVOIRS POPULAIRES) }\end{array}$ & $\begin{array}{l}\text { Pensée collective, } \\
\text { Weltanschauung }\end{array}$ \\
\hline
\end{tabular}

Source: Propre élaboration.

Sous tous les cieux, ce sont des élites qui produisent les spéculations et les savoirs du niveau-A. Leurs productions ne relèvent pas du sens commun ni de la pensée populaire, encore moins de «l'impensé collectif». Les savants des sociétés africaines traditionnelles ont construit «une explication [...] des manifestations de la nature (anthropologie, botanique, zoologie, géologie, astronomie, anatomie et physiologie) comme des faits sociaux (structures sociales, religieuses et politiques, techniques, arts, économie etc.)» (DIETERLEN, 1959, p. 24). Ces connaissances ne sont accessibles qu'à des initiés d'un certain rang. On y a accès progressivement, par degrés. Dans les sociétés peul par exemple, on compte «trente-trois degrés [d'instruction] auxquels s'ajoutent trois degrés supérieurs» (BÂA HAMPÂTÉ; DIETERLEN, 2009, p. 19). Les dogons, quant à eux, distinguent quatre paliers du savoir (cfr. GRIAULE, 1952, p. 27): au niveau inférieur, les savoirs relevant de la catégorie dite "giri so»: légendes populaires, affabulations, explications simplifiées; au deuxième niveau, les savoirs de la catégorie dite «benne so»: exposition des grandes divisions du savoir, sans une explication détaillée; puis, les savoirs de la catégorie "bo loso»: approfondissement des savoirs de la deuxième catégorie; enfin, les savoirs de la catégorie «so dayi»: exposition systématique de «l'édifice du savoir 
dans sa complexité ordonnée». Les cosmo-théosophies appartiennent à cette catégorie.

Griaule note que So dayi, l'ultime état de la connaissance

Ne s'acquiert qu'après de longues années d'application et de persévérance. Le fait de posséder des principes généraux et des procédés de calcul très secrets ne suffit pas à un homme pour qu'on le dise détenteur de la «parole claire» (so dayi), il faut connaître matériellement tous les détails qui s'y rapportent. C'est ainsi que le système de signes graphiques, qui en comporte des milliers, doit être théoriquement possédé dans son entier et non pas seulement connu dans sa structure et dans son fonctionnement. (GRIAULE, 1952, p. 29).

II n'est pas rare que, dans une même aire culturelle, cohabitent différentes Ecoles Initiatiques, avec des conceptions théoriques parfois convergentes, parfois divergentes. Ainsi, distingue-t-on, par exemple, divers courants de pensée autour des savoirs du corpus Mvett. ${ }^{1}$ Tsira Ndong Ndoutoume, un des brillants érudits de cette tradition séculaire, se réclame de l'Ecole du Vénérable Zue-Nguema: "Cette connaissance de l'univers, je l'ai reçue, voilà quatre décennies et par enseignement direct, de mon Maître Père Zue-Nguema, qui la détenait lui-même exactement de la même manière des Maîtres qui l'avaient précédé» (NDOUTOUME, 1993, p. 27-8). L'auteur établit la généalogie des maîtres de cette école et des affiliations qui les lient dans l'ordre initiatique. Cette généalogie remonte, par delà le temps et l'espace, à un Grand Maître mythique, Oyono Ada Ngone, "I'homme qui entendit premièrement la voix du Verbe» (NDOUTOUME, 1993, p. 12). Grégoire Biyogo distingue «cinq courants de mvett»: Le courant spiritualiste, le courant ontologique, le courant historique et sociopolitique, le courant encyclopédique, le courant du comparatisme historique. La diversité de ces courants atteste qu'il existe des «nuances de pensée» entre les diverses écoles cosmo-théosophiques bien qu'elles se réclament d'un même «imaginaire civilisationnel».

On a souvent cherché à lire obstinément les textes du niveau-A seulement sous l'angle religieux (Niveau-B). Théophile Obenga fustige cette

\footnotetext{
${ }^{1}$ Le "Mvett" est une tradition cosmo-théosophique Fang ou Ekang (Gabon, Cameroun, Guinée Equatoriale).
} 
tendance chez les commentateurs des textes du corpus égyptien:

C'est l'erreur des exégètes d'avoir interprété tous les textes importants égyptiens comme des documents religieux, laissés par leurs auteurs pour comprendre leur religion. De l'Egypte antique on ne parlera que de «religion» jamais de «philosophie»: le sort est imputable aux seuls lecteurs des textes égyptiens. Les égyptologues africains doivent réagir contre cette tendance généralisée qui peut tenir d'un préjugé inavoué cependant dangereux. Les anciens Egyptiens ont pensé l'être, la vie, la mort, etc. Ne réduisons plus leurs écrits importants à la seule dimension «sacrée», «religieuse». (OBENGA, 1990, p. 61).

Comme le montre notre tableau, la pensée religieuse, en tant que système de croyances, de valeurs et de pratiques rituelles, dérive de la pensée cosmo-théosophique. Elle n'est donc pas la référence première.

Les auteurs des cosmo-théosophies africaines adoptent généralement un schéma explicatif qui peut être résumé comme suit (cfr. CANEVA; SINSIN; THURUTHIYIL, 2017, p. 169):

Tableau 3-Structure du modèle explicatif des cosmo-théosophies africaines

\begin{tabular}{|l|l|l|l|}
\hline \multicolumn{3}{|c|}{ Le monde, le cosmos ou l'univers } \\
\cline { 2 - 4 } \multicolumn{2}{|c|}{ Origine } & \multicolumn{2}{|c|}{ Structure et composition } \\
\cline { 2 - 4 } & $\begin{array}{l}\text { Qui «pense» le monde } \\
\text { (le projette } \\
\text { intentionnellement) }\end{array}$ & $\begin{array}{l}\text { Une forme primordiale: } \\
\text { un œuf, une spirale, un } \\
\text { flux etc. }\end{array}$ & $\begin{array}{c}\text { Principes } \\
\text { structurels }\end{array}$ \\
\cline { 2 - 4 } $\begin{array}{l}\text { Unité } \\
\text { Créateur }\end{array}$ & $\begin{array}{l}\text { Puis, le façonne avec } \\
\text { la Parole, le Souffle et } \\
\text { le Geste }\end{array}$ & $\begin{array}{l}\text { Les 4 Eléments (Eau, } \\
\text { Terre, Feu, Air) }\end{array}$ & $\begin{array}{l}\text { Multiplicité, } \\
\text { diversité, dualité, } \\
\text { complémentarité }\end{array}$ \\
\cline { 2 - 4 } & $\begin{array}{l}\text { Le conserve et } \\
\text { le renouvelle } \\
\text { continuellement } \\
\text { (Création continue) }\end{array}$ & Temps, Espace, Esprit & $\begin{array}{l}\text { Rythme, Equilibre, } \\
\text { Harmonie, } \\
\text { Intelligibilité. }\end{array}$ \\
\hline
\end{tabular}

Source: Caneva, Sinsin, Thuruthiyil (2017, p. 169).

Les deux colonnes principales de ce tableau indiquent que les cosmothéosophies sont à la fois des cosmogenèses (tentatives d'explication de 
l'origine du monde) et des cosmologies (tentatives d'explication de la structure du monde). Ce sont des «systèmes d'intelligibilité du réel» (BA MOCTAR, 2007, p. 241). Nous tenterons d'élucider ce schéma explicatif à partir d'un corpus de fragments de textes ou de péricopes.

\section{NǓDÒ: L'ONTOGENÈSE OU L'ORIGINE DES CHOSES}

$[\mathrm{T}-\mathrm{I}]$

"Je naquis dans le Noun

Avant que le ciel ne vint à l'existence

Avant que la terre ne vint à l'existence

Avant que ce qui devait être établi ferme ne vint à l'existence».

(«Textes des Pyramides», 1040, apud OBENGA, 1990, p. 29).

$[\mathrm{T}-\mathrm{II}]$

"Je suis Atoum quand je suis seul à exister, étant seul dans le Noun». («Livre pour sortir au jour», apud OBENGA, 1990, p. 38).

[T-III]

"Je fis tout ce que je fis, étant seul, avant que personne d'autre (que moi) ne se fût manifesté à l'existence, pour agir en ma compagnie en ces lieux. Je fis les modes d'existence à partir de cette force (qui est en moi)».

( LLivre de connaître les modes d'existence de Ra», apud OBENGA, 1990, p. 56).

[T-IV]

«Au commencement de Toutes-les-Choses, l'Esprit Aîné, Maweeja Nnangila, le premier, l'aîné et le grand seigneur de tous les Esprits qui apparurent par la suite, se manifesta, seul, et de par soi-même». («Cosmogonie bantu», FOURCHE; MORLIGHEM, 2002, p. 31).

$[\mathrm{T}-\mathrm{V}]$ «Suivant leur sorte, il les créa en usant de l'un ou l'autre de ses trois grands pouvoirs de science secrète:

L'Emission du Verbe

L'Appel par le Geste

Le Souffle».

(«Cosmogonie bantu», FOURCHE; MORLIGHEM, 2002, p. 53). 


\begin{abstract}
$[\mathrm{T}-\mathrm{VI}]$
«Au commencement Eyô est le seul à être. Il est seul. La vie, la lumière sont en lui. II en jouit seul. Tout, autour de lui, est néant. II n'y a pas de tam ou eyong (temps) ni d'évuigne ou lamé (espace), ni de megnoung (matière). Il est seul et seul. Mais il est. II est hors de tam, de lamé et de megnoung. Tout se réduit à lui. II se met à réfléchir. Il pense et, de sa seule intelligence (ossimann) trouve le mot vie. Donc il vit. Eyô est le seul à vivre, le seul à être. Il se dit: 'Et si j'élargissais la vie'». («Texte Mvett», trad. NDOUTOUME, 1993, p. 23).
\end{abstract}

\title{
Analyse
}

Les auteurs de ces textes postulent un Principe Premier, Raison Suprême, Fondement et Origine des choses. Les séquences (T-I) et (T-II) indiquent que ce Principe se manifeste à l'intérieur d'une forme primordiale appelée «Noun». Le Noun, écrit Obenga (1990, p. 30), c’est «Cela qui ne ressemble à rien de connu, d'édifié», mais qui contient déjà «toute la matière première qui va être mise en œuvre par le démiurge». Le philosophe congolais confond le Noun avec la Matière: «L'Idée sort, puissante, de la Matière brute. Au commencement, il y a la matière, une eau faible, obscure, abyssale, mais puissante, dynamique» (OBENGA, 1990, p. 31). Obenga semble se contredire car, après avoir énoncé que le Noun «ne ressemble à rien de connu», il nous le présente comme étant de la Matière. Cheikh Moctar Ba (2007, p. 72-3) tombe, lui aussi, dans la même ambigüité. D’une part, il conçoit le Noun comme une «béance», un «état originel», un «ensemble de possibilités existentielles, en somme, comme une réalité non matérielle; de l'autre, il le décrit comme une " eau primordiale», une "étendue aqueuse» (distincte cependant du Chaos des mythologies grecques). Comment le Noun peut-il être considéré comme «matière» si «tout ce qui devait être établi ferme» (T1) ne viendra à l'existence que par la suite, avec l'acte créateur du Démiurge? Les savants égyptiens utilisaient certes la métaphore du "flux" ou du "filet d'eau" pour exprimer le concept du Noun (voir le signe N35A de la liste Gardiner), mais ce n'était là qu'une représentation idéographique. L’idée d’une «matière primordiale incréée» est étrangère à la pensée nilotique. Le déterminatif figuratif qui accompagne la graphie du concept 
suggère à notre avis l'idée d'une continuité atemporelle, d'un écoulement sans commencement ni fin, au-delà du temps et de l'espace; en d'autres termes, elle suggère l'idée d'une infinitude absolue. Pour Cheikh Anta Diop, la philosophie du Noun a comme corollaire la thèse de l'éternité de l'Etre et celle de l'impossibilité du non-être: «Il n'a pas existé dans la cosmogonie égyptienne un instant zéro, à partir duquel l'être, la matière surgit du néant, du non-être ; l'être, au sens de Heidegger et de J-P. Sartre, est éternel ; sa plénitude exclut à priori la possibilité même hypothétique du non-être, du néant comme absurdité suprême» (DIOP, 1981, p. 131).

Les textes de ce premier corpus mettent en évidence l'Unicité absolue du Démiurge. II créa seul dans le Noun. Nous sommes ici en présence d'une pensée radicalement monothéiste dans son énonciation première. Le Créateur (Atoum, Maweeja Nngangila, Eyô) reste l'Unique Origine de Soi-même et de tout ce qui est. Une certaine exégèse des fragments (T1) et (T2) pourrait conclure à un dualisme originel Noun-Atoum. Ce serait une erreur, car le Noun est consubstantiel au Créateur et forme avec lui une seule Entité, Indivisible, Incréée, Invisible. Le Noun est la béance insondable de l'Etre Suprême (Atoum).

Les textes tentent par ailleurs de décrire la manière dont l'Etre-Un créa les modes d'existence. II les créa usant de la "force qui est en lui» (T-III), c'est-à-dire la force de la «pensée, de l'intelligence» (T-VI) ou encore la puissance du Verbe, du Geste et du Souffle (T-V). Elloué-Engoune (2008, p. 24), commentant les textes mvett, écrit: «ll a conçu la création dans Sa Pensée et la réalisa par Son Verbe». Les Ecoles dogon enseignent la même doctrine: «Amma (Dieu) maintenait l'ensemble car il avait tracé en luimême le plan du monde et de son extension. Car Amma a dessiné l'univers avant de le créer. [La création par la Pensée]». Puis, «les signes d’Amma, qu'il a envoyés dans le monde, sont allés, sont entrés dans les choses, qui, (à ce moment) furent. [La création par l'expression graphique du Verbe]» (GRIAULE ; DIETERLEN, 1991, p. 63-4 ; p. 71-2). Ainsi, «au commencement était la Raison, ensuite seulement le Verbe. Avant de faire être concrètement en prononçant le nom [...] même de ce qui est appelé à être, le démiurge conçoit d'abord cela qui va être par la puissance du verbe, l'efficience de la parole créatrice» (OBENGA, 1990, p. 59-60). 


\section{NǓTǏN, NǓNYÍ, NǓDANNǓ: L'EXISTENCE, L'ESSENCE ET LE MOUVEMENT}

[T-VII]

"Quand je me suis manifesté à l'existence, l'existence exista. Je vins à l'existence sous la forme de l'Existant qui est venu à l'existence, en la Première Fois. Venu à l'existence sous le mode d'existence de l'Existant, j'existai donc. [...] Une foule de modes d'existences vinrent à l'existence dès ce début».

(«Livre de connaître les modes d'existence de Ra», apud OBENGA, 1990, p. 56-7).

[T-VIII]

«Créant ainsi Toutes-les-choses [...], Maweeja Nnangila leur conférait du même coup leurs qualités primordiales».

(«Cosmogonie bantu», in FOURCHE; MORLIGHEM, 2002, p. 39).

$[\mathrm{T}-\mathrm{IX}]$

"Ces qualités apparaissent aux initiés d'après les modes d'action des choses auxquelles elles appartiennent».

(«Cosmogonie bantu», in FOURCHE; MORLIGHEM, 2002, p. 47)

\section{Analyse}

Alexis Kagame utilise la notion d'existant pour désigner le concept d'être tel qu'il est conçu dans les cosmo-théosophies bantu. Par ce terme, il entend «ce qui est réalisé dans la nature et ce qui peut l'être, chacun dans son ordre, soit en lui-même (substance), soit par adhésion à la substance (accidentalité) et intemporellement» (KAGAME, 1976, p. 117). Dans la philosophie occidentale classique, «l'être en lui-même» est appelé «essence». L'essence, c'est «ce qui est considéré comme formant le fond de l'être, par opposition aux modifications qui ne l'atteignent que superficiellement ou temporellement». C'est aussi «ce qui constitue la nature d'un être, par opposition au fait d'être» (LALANDE, 1996, p. 301). Le «fait d'être» est ce qui se nomme «existence».

Partant de ces définitions, Ernest Menyomo (2010, p. 100) se demande si dans l'ontologie africaine l'être de l'essence appartient à l'essence 
elle-même ou à l'existence. Si l'essence est créée, répond-il, "elle l'est nécessairement dans un temps et par là, elle n'est plus essentielle, d'où il appert que l'essence de l'essence est de fuir l'essence pour entrer dans l'existence, c'est-à-dire que l'essence est avènement, tandis que l'existence est nécessairement évènement». L'auteur ne précise pas le sens qu'il donne à ces deux concepts, "avènement" et "évènement". II pose ensuite un second problème qui n'est pas nouveau: dans la pensée négro-africaine, l'essence précède-elle l'existence ou est-ce l'inverse? II cite Marcien Towa, lequel se fonde sur la péricope T-VII pour défendre la thèse de l'antériorité de l'existence. ${ }^{2}$ Au fond, à y voir de près, il ne nous semble pas fondé de s'appuyer sur T-VII pour défendre l'idée d'une prééminence de l'existence sur l'essence, ni l'inverse. Les deux principes sont inséparables. C'est dans un même acte que l'Etre (Nǔ) se manifeste (Nǔtǐn) comme étant l'Existant (Nǔnyí): «Dès qu'il existe, l'Existant amène à l'existence l'existence: cela de façon immédiate, une sorte d'épiphanie soudaine de l'être dans sa manifestation même» (OBENGA, 1990, p. 59). L'existence présuppose l'essence et vice versa. La péricope T-VIII exprime cela de manière explicite: en créant les choses (existence), Maweeja Nnangila leur confère «du même coup» leurs qualités (essence). L'expression «du même coup» a ici tout son sens.

Une troisième problématique retient l'attention du philosophe camerounais: le rapport entre «essence» et «identité»:

Pas plus que l'essence, l'identité ne sera au départ, mais seulement à l'arrivée. L'identité est résultat, c'est l'ensemble de toutes les variations que l'on aura acquises, sans que pour autant ces transformations excluent d'autres transformations futures. L'identité n'est pas séparable de la diversité, en ce sens que la variation à laquelle elle est soumise lui donne la possibilité d'être elle-même et autre qu'elle-même. (MENYOMO, 2010, p. 100-1).

\footnotetext{
${ }^{2}$ Towa se dresse en outre contre l'idée d'une essence «éternitaire», immobile, absolue, parfaite. Il craint le totalitarisme et le dogmatisme qui peuvent en découler: «Aussitôt que l'omniscience et la perfection d'un être réel sont posées, sont aussi par là même posés en principe le dogmatisme et l'enfermement, d'autant plus que les prétendues omniscience et perfection ne sont invoquées que pour justifier l'absolutisme socio-politique effectif ou potentiel» (TOWA, 1979, p. 39). Cette position est fort discutable. II n'est nullement établi que l'absolutisme soit nécessairement lié à l'idée d'une essence omnisciente et parfaite.
} 
Selon Menyomo, l'essence n'est jamais quelque chose d'immuable ni d'achevé. Elle est "prétention à être», tension continue vers l'accomplissement. II s'ensuit que «l'essentiel est le mouvement, l'essentiel est le transitoire». Précisons cependant que l'accomplissement n'est pas une mutation d'essence, mais une actualisation des qualités potentielles dont l'essence est porteuse. Ces qualités potentielles constituent ce que les maîtres Ciluba appellent «qualités de création». Si l'accomplissement n'est pas une mutation substantielle ou essentielle, comment expliquer par exemple le processus de divinisation de l'Homme, dont il est fait mention dans la plupart des cosmo-théosophies africaines ? Comment l'homme peutil devenir dieu ? La réponse, c'est que, selon les textes mêmes, I'homme devient dieu tout simplement parce qu'il est dans son essence de le devenir. On ne saurait développer ici ce thème, car cela nous éloignerait de notre sujet.

Revenons donc au rapport essence/identité. Du point de vue anthropologique, il y a une distinction entre les deux notions. L'essence de l'homme, c'est l'ensemble des qualités de création qui le constituent comme membre d'une espèce spécifique: qualités génériques ou communes (corporéité, rationalité, émotivité, etc.) et qualités individuelles potentielles (talents, sensibilité, goûts, etc.). L'identité, par contre, c'est, d'une part, la découverte ou la prise de conscience de l'essence humaine individuelle et collective, et, d'autre part, la mobilisation des qualités potentielles de l'essence pour une cause finale: l'accomplissement. En d'autres termes, l'essence est donnée (ensemble des qualités de création), tandis que I'identité est une construction permanente (actualisation continue des qualités potentielles de création).

Existence, Essence et Identité s'inscrivent dans la dynamique du Devenir. En effet, toute la réalité créée est gouvernée par la loi du mouvement et de l'évolution. Cette loi ou dynamique s'entend de deux manières: a) comme re-création ou création continue. Ainsi, pour Moctar Ba (2007, p. 249), l'idée de Noun, exprimant une infinité de possibilités potentielles, «permet d'inscrire l'avènement de l'Etre dans une création continuée, marquée par une série de mouvements et de ruptures dont le sens est de favoriser la venue à l'existence de nouveaux êtres» ; b) comme 
passage d'un état-x à un état-y à partir d'un principe interne: "L'idée de "principe interne" rend compte de la présence de la loi du devenir comme force de mouvement, de changement, jouissant d'une transcendance au processus existentiel» (BA MOCTAR, 2007, p. 249). C'est la loi du devenir et du mouvement qui rend possible l'actualisation des formes existentielles potentielles.

Certains textes indiquent que le Créateur, non seulement est la source primordiale de tout mouvement, mais est lui-même mouvement et expression suprême du devenir. Griaule et Dieterlen (1991, p. 106) traduisent un texte dogon où on lit ceci: «Amma, à l'intérieur de l'œuf, était lui-même comme un mouvement spiralant». Citons par ailleurs cet extrait du Livre de la sortie au jour (improprement appelé "Livre des morts") où I'Etre-Un proclame: "C'est moi le Devenir de Khepra, lorsque devint pour moi le Devenir des Devenirs après mon Devenir» (cit. in DIOP, 1981, p. 413). En d'autres termes, au commencement (pour ainsi dire), I'Etre-Un devient. ${ }^{3}$ Puis, de lui, procède le «Devenir des Devenirs», c'est-à-dire toute la chaîne des autres devenirs. Moctar Ba (2007, p. 250) interprète le Premier Devenir, celui de l'Etre-Un, comme passage «de la somnolence à la manifestation concrète ou expressive». Vu que la notion de «somnolence» peut prêter à confusion, nous préférons, pour notre part, parler tout simplement de «passage de la non-manifestation à la manifestation».

\section{NǓĐÒKPJ́: UNION ET PARENTÉ DES ÉLÉMENTS}

$[\mathrm{T}-\mathrm{X}]$

«Dans le principe, alors que Maweeja Nnangila était encore seul, tout était Un, entier comme l'est un œuf, entier comme l'est une

\footnotetext{
${ }^{3}$ Nous utilisons ici le verbe devenir comme un verbe intransitif pour traduire littéralement le terme «kheper» employé dans le texte original. A propos de ce concept, Moctar Ba (2007, p. 250) note: «En indiquant sa première manifestation par l'usage de l'expression "Kheper" ("venir à l'existence"), qui, selon Obenga, est un «verbe intransitif» ayant, entre autres, le sens de "changer», "transformer», Râ (autre nom du Dieu suprême) ouvre la possibilité d'inscrire le Devenir au cœur du processus existentiel. [...] Ainsi, Râ jouit-il d'un principe de mouvement ou de changement in agente lui permettant de Devenir ce qu'il doit être par le concours de sa propre force ou de son pouvoir de transformation».
} 
calebasse».

(«Cosmogonie bantu», in FOURCHE; MORLIGHEM, 2002, p. 32).

[T-XI]

"L'eau, semence divine, pénétra donc au sein de la terre et la génération poursuivit le cycle régulier de la gémelliparité.

[...] L'humidité est dans tout».

(«Entretiens avec Ogotemmêli», in GRIAULE, 1966, p. 16-7).

\section{Analyse}

Ces courts extraits indiquent que l'univers est une totalité (un "œuf», une "calebasse») et que tous les êtres sont reliés entre eux par un principe ontologique universel que le Vénérable Ogotemmêli désigne par le concept d'eau. Quand Thalès de Milet annonce que "tout est eau», il ne fait que proclamer ce principe d'unité originelle: "Thalès, écrit Nietzsche, a vu l'unité de l'être, et quand il a voulu la dire, il a parlé de l'eau» (cit. in OBENGA, 2005, p. 24). C'est à peu près la même intuition qu'on retrouve dans l'enseignement du vénérable sage dogon. En commentant la péricope T-XI, Mbongo (2013, p. 50) écrit:

Nous ne sommes pas ici dans une spéculation inintelligible [...]. Le concept est présent et se sait rationnel: le concept d'eau. Dans le fouillis des êtres et des choses, la pensée du vieux sage dogon va à la recherche d'un élément et d'un seul, qui serait l'entité essentielle rendant compte de tout le reste: le principe des choses et des êtres. Cette place revient à la fluidité agissante d'un liquide «pur», l'eau.

Obenga souligne, lui aussi, la valeur éminemment philosophique de cette idée de l'eau conçue comme principe de l'unité primordiale de la totalité. Elle remonte, selon lui, à l'antiquité pharaonique: «L'eau de Thalès, c'est exactement le Noun égyptien, les eaux primordiales, avec toute la force d'un concept concret». II ajoute:

L'Egypte, bien avant Thalès, a posé les concepts de Noun et de Râ, l'unité de tout comme une unité vivante et comme "divine" à la fois. Ce discours qui fait partie lui-même du tout cosmique, est proprement philosophique, car l'opinion commune égyptienne était certainement assez éloignée de telles conceptions sur la réalité 
de l'univers. Les prêtres égyptiens ont donc enseigné à Thalès une découverte essentielle: le rapport entre "l'esprit» et toutes choses, en reconnaissant dans l'eau «l'origine» et la "condition première» de tout ce qui est: le Noun est radicalement un principe d'unité. (OBENGA, 2005, p. 25).

Menyomo (2010) tire deux conclusions du postulat de l'unité originelle des éléments:

Matière et esprit sont liés

La parenté n'est pas seulement un fait social ; elle est d'abord une structure ontologique.

\title{
6 NǓVOVO, NǓGBJNVO: DIVERSITÉ ET DISTINCTION
}

\author{
$[\mathrm{T}-\mathrm{XII}]$
}

"Je suis celui qui a séparé ce qui était réuni ; j’ai circulé autour de son CEuf. Je suis le matin du temps».

(«Livre de la sortie au jour», apud OBENGA, 1990, p. 44).

$[\mathrm{T}-\mathrm{XIII}]$

«Lorsque Aki Ngoss éclata, donnant naissance à Lamé (espace), et que Lamé s'étendit devenant Ndalamé (infiniment grand), Eyô fit la Différence (Nkanané). Dans l'immensité il fit apparaître d'autres formes de vie. Ainsi les ténèbres alternèrent avec la lumière, le laid avec le beau, la nuit avec le jour, le dur avec le tendre, le saisissable avec l'insaisissable, le non avec le oui, le mal avec le bien, la mort avec la vie».

(«Texte Mvett», trad. NDOUTOUME, 1993, p. 27).

[T-XIV]

«Aki Ngoss [l'CEuf Cosmique] grossit démesurément comme une boule de caoutchouc qu'on gonfle, devint incandescent, explosa ( $a l a^{\prime} a$ ) en d'infinies particules (megnoung) étincelantes et donna naissance à: Mikour mi Aki ou MikourNegnoung:

Les brouillards issus d'Aki Ngoss: Galaxies;

Mikour mi Aki œuvra et engendra

Biyem-YemaMikour:

Les Vides issus des Brouillards, Vides intergalactiques;

Biyem-Yema œuvra et engendra 


\section{Dzop Biyem-Yema:}

Le Ciel issu de Biyem-Yema, des Vides;

Dzop œuvra et engendra

Bikoko bi Dzop:

Les nébuleuses, les constellations».

(«Texte Mvett», trad. NDOUTOUME, 1993, p. 17-8).

[T-XV]

«ll créa donc d'abord, et de cette façon, par paires jumelles:

L'énergie et le Souffle

L'Eau et le Feu

Le Ciel du Sommet et la Terre

La Lumière et les Ténèbres

Les deux Grands luminaires (le Soleil et la Lune) et les étoiles».

(«Cosmogonie bantu», in FOURCHE; MORLIGHEM, 2002, p. 37).

$[\mathrm{T}-\mathrm{XVI}]$

«Maweeja Nnangila, en même temps qu'il créa chaque chose, leur conférait son nom, et du même coup, les qualités que signifiait son nom».

(«Cosmogonie bantu», in FOURCHE, MORLIGHEM, 2002, p. 43).

\section{Analyse}

Affirmer l'unité ou la parenté de l'esprit et de la matière ne signifie pas défendre du coup une position animiste. Menyomo n’a pu éviter cette confusion:

Si l'animisme syncrétique est étudié avec sérieux, sans préjugés, on peut y déceler une philosophie embryonnaire, où l'être apparaît essentiellement indéterminé, les substances mêlées les unes aux autres [...]. La confusion qui frappe les êtres et les choses ne doit pas être interprétée de manière négative, au contraire, c'est de la confusion que doit jaillir la lumière qui distingue les choses. (MENYOMO, 2010, p. 28).

Deux remarques s'imposent: (a) l'être, pris dans sa totalité, est matière et esprit, mais cela n'induit nullement l'idée que tous les étants soient des esprits ou qu'il y ait autant d'esprits qu'il y a d'objets. La pensée religieuse africaine postule l'existence d'esprits tutélaires qui veillent sur les Quatre 
Eléments Primordiaux (Eau-Terre-Air-Feu), la Faune et la Flore. Souvent, une interprétation superficielle et mécaniciste confond ces esprits tutélaires avec les entités placées, pour ainsi dire, sous leur "tutelle protectrice". De là dérive le présupposé d'un prétendu «animisme» qu'on colle indûment à la spiritualité africaine (Voir EFFA, 2015). (b) L'union originelle ou la parenté des êtres matériels et spirituels n'implique aucune idée de confusion ou d'indistinction. Ici aussi, Menyomo semble entretenir le flou: «Au départ, écrit-il, la pensée négro-africaine estime toutes les choses confondues». Affirmation pour le moins discutable. Avant l'actualisation du plan de la création, les choses appelées à exister sont bien distinctes dans la pensée du Créateur. Commentant T-VII, Obenga (1990, p. 59) écrit: «Quand la raison [divine] a tout conçu, le plan de la création se présente devant l'Un-Créateur, devant sa face [...], en toute visibilité, sans confusion. La création est une idée claire, nette, distincte, consistante chez le Créateur, lui qui est absolu, amour, volonté et raison, efficacité par excellence, maître de la totalité».

L'éclatement de «l'œuf primordial» (T-XIV) matérialise cette distinction des choses et imprime dans l'ordre de la création le principe de leur séparation ou différentiation (Nkannané). Chaque chose a son essence propre, son «nom» (T-XVI) et appartient à une catégorie ontologique précise.

Alexis Kagame distingue divers types d'existants dans les cosmothéosophies bantu: le Pré-existant, l'existant figé, l'existant assimilatif, l'existant sensitif, l'existant raisonnable. A l'exception du Pré-existant, tous les existants sont appelés «Ntu», (un terme lexicalement et sémantiquement proche du Nǔ en fongbe):

Tout ce qu'il est possible de concevoir et d'exprimer (substance, accident, nombre, etc.) est quelque chose, une entité, un NTU, mais chaque entité dans son ordre [...]; c'est dans ce sens que le genre suprême [NTU] est dit applicable à toute entité d'une manière analogique, et pas univoque (signifiant la même chose en chaque chose). (KAGAME, 1976, p. 121).

Les entités NTU se répartissent en quatre catégories métaphysiques que nous nous schématisons comme suit: 
Tableau 4 - Les catégories métaphysiques des entités Ntu

\begin{tabular}{|c|c|c|c|}
\hline $\begin{array}{c}\text { Catégories } \\
\text { métaphysiques }\end{array}$ & Définition & Spécifications & Illustrations \\
\hline \multirow[b]{2}{*}{ MU-NTU } & \multirow{2}{*}{\begin{tabular}{|l|} 
l'existant doué \\
d'intelligence
\end{tabular}} & Masculin & Homme \\
\hline & & Féminin & Femme \\
\hline \multirow{3}{*}{ KI-NTU } & \multirow{3}{*}{$\begin{array}{l}\text { L'existant sans } \\
\text { intelligence } \\
\text { (Chose) }\end{array}$} & Figé (inanimé) & Le minéral \\
\hline & & Assimilatif (inanimé) & Le végétal \\
\hline & & $\begin{array}{l}\text { Sensitif (doué du } \\
\text { principe vital) }\end{array}$ & L'animal \\
\hline \multirow{2}{*}{ HA-NTU } & \multirow{2}{*}{$\begin{array}{l}\text { L'existant } \\
\text { localisateur }\end{array}$} & $\begin{array}{l}\text { Sur la dimension } \\
\text { espace }\end{array}$ & Lieu \\
\hline & & $\begin{array}{l}\text { Sur la dimension } \\
\text { durée }\end{array}$ & Temps \\
\hline \multirow[b]{2}{*}{ KU-NTU } & \multirow[b]{2}{*}{$\begin{array}{l}\text { L'existant } \\
\text { modal }\end{array}$} & Interne & Quantité, Qualité \\
\hline & & $\begin{array}{l}\text { Externe } \\
\text { (Relation) }\end{array}$ & $\begin{array}{l}\text { Action, Passion, } \\
\text { Position, Possession, } \\
\text { Circumposition, } \\
\text { nombre (succession- } \\
\text {-juxtaposition) }\end{array}$ \\
\hline
\end{tabular}

Source: Propre élaboration.

La différentiation des NTU présuppose l'existence du «Rien», lequel se distingue du «Néant». Alors que le Néant est «inconcevable», le Rien est «le principe de la distinction entre les existants matériels et la cause du multiple. Deux objets sont deux, parce qu'entre eux il y a le rien» (KAGAME, 1976, p. 128). Le rien existe, mais il est indéterminé et indéterminable; il est simplement un «vide diviseur».

A propos du Pré-existant, l'auteur écrit:

Ce tout premier qui jouit de l'exister sans l'avoir reçu, en qui est fondée l'explication ultime des commençants-à-exister, sera, sans ajoute, le Pré-existant. Comment les créateurs de la Culture Bantu ont-ils perçu et exprimé cette vérité fondamentale? Ils l'ont fait par exclusion dans la formulation des catégories des existants. (KAGAME, 1976, p. 130).

Notons, pour conclure, que la différentiation ne dissout pas le lien originel ou la parenté ontologique qui lie les existants. 


\section{LE RÉALISME SIGNIFICATIONNEL}

Quelle vision métaphysique dominante émerge des cosmo-théosophies étudiées? Idéalisme, matérialisme, monisme, dualisme? Les avis des philosophes africains divergent sur cette question. Pour Kwame Nkrumah, l'ontologie africaine se fonde sur un postulat matérialiste qu'il résume comme suit: «Notre univers est un univers naturel. Et son fondement est la matière, avec ses lois objectives». II ajoute: «la transformation dialectique de la matière est ce qui sert de fondement à la possibilité de l'évolution des espèces» (NKRUMAH, 1976, p. 37-41). II s'agit cependant d'un matérialisme non athée car il reconnaît l'existence de l'esprit comme une catégorie d'être émanant dialectiquement de la matière: «Strictement parlant, l'affirmation de la seule réalité de la matière est un athéisme» (NKRUMAH, 1976, p. 105).

En s'appuyant sur une certaine interprétation des cosmo-théosophies bantu, Alexis Kagame défend par contre la thèse d'une ontologie immatérialiste qui, selon lui, s'enracine dans la conception même du Divin:

[Des] idées principales que les initiateurs bantu ont formulées dans les noms désignant le Pré-existant, ou l'Existant Eternel, celle logiquement antérieure à toutes les autres et qui sert de fondement est la Transcendance du Préexistant [...]. Il est analogue aux esprits désincarnés; il est par conséquent incorporel, d'où il est corollairement Immatériel (Invisible, Spirituel) et immortel. (KAGAME, 1976, p. 151-2).

Léopold Sédar Senghor (1977, p. 71) propose quant à lui une approche prétendument moniste:

L'étoffe de l'univers n'est pas composée de deux, mais d'une seule réalité sous la forme de deux phénomènes; il n'y a pas la matière et l'énergie, pas même la matière et l'esprit, mais l'esprit-matière comme il y a l'espace-temps. La matière et l'esprit se réduisent, l'une à l'autre, à un «tissu de relations», pour parler comme Bachelard: à l'énergie définie comme un réseau de forces. II n'y a donc, dans la matière-esprit, qu'une énergie unique, qui se présente sous deux aspects. L'une, l'énergie tangentielle, celle du «dehors», est matérielle et quantitative. [...] L'autre, l'énergie radiale, celle du «dedans», est psychique et qualitative.

Nsame Mbongo (2013, p. 125) soutient, pour sa part, la thèse d'un naturalisme significationnel à base matérialiste: 
A notre sens, le fondement de la philosophie classique négro-africaine relève du matérialisme et non de l'idéalisme ou de la prétendue troisième voie énergétique. C'est ce matérialisme philosophique, qui est en même temps dialectique, que nous appelons naturalisme significationnel ou significationnisme.

Que penser de telles positions? A la lumière des fragments que nous avons examinés, la thèse matérialiste ne nous semble pas fondée. Pour justifier cette thèse, Mbongo se réfère au concept de Noun en l'interprétant dans une perspective purement naturaliste: «La matière aquatique (l'eau) apparaît comme l'élément premier de l'existence» (MBONGO, 2013, p. 126). Comme nous l'avons indiqué plus haut, cette interprétation littérale ne rend pas compte de la richesse et de la profondeur du concept. Elle rencontre surtout une objection fondamentale: si la matière est l'élément premier, comment s'explique l'émergence de l'esprit ou de la conscience? Nkrumah propose la théorie de la «conversion catégorielle». Mbongo reprend sous une certaine forme cette théorie: "L'Etre en tant que Matière initiale inconsciente prend ensuite une dimension consciente, devenant lucide, capable de faire des plans et des projets pour conduire l'œuvre de création» (MBONGO, 2013, p. 127). Par quelle alchimie ou quel processus la matière inconsciente devient-elle consciente par son seul et propre mouvement? Quelles sont les lois objectives et dialectiques de la matière qui rendent possibles cette conversion? Nos auteurs peinent à résoudre cette équation. Mbongo avance une explication ingénieuse, qui, loin de résoudre l'énigme, ne fait que l'étaler de manière plus éclatante. Il y a, selon lui, trois «modes» à travers lesquels la matière produit de l'immatériel:

1. L'évolution ascendante (l'auto-dynamisme contradictoire de la matière fonctionne selon une logique assurant le passage du simple au complexe, du quantitatif au qualitatif, de l'inférieur au supérieur, à travers la complexification, la diversification et le perfectionnement progressifs); 2. L'organisation cohérente (la matière n'existe pas de manière chaotique, mais se constitue comme construction [...]); 3 . La dimension axiologique (la matière la plus évoluée se convertit en réalité capable de sensibilité esthétique, de sens moral, de conscience intellectuelle. (MBONGO, 2013, p. 132). 
Nkrumah évite de donner des explications aussi apodictiques: «Ce n'est pas la tâche de la philosophie que d'indiquer les détails d'une telle conversion catégorielle, c'est l'une des tâches de la science; la philosophie n'intervient que montrer la possibilité de la conversion» (NKRUMAH, 1976, p. 31-2). La science pourra-t-elle vraiment nous fournir un jour des réponses quant à ces «détails»?

Venons-en au monisme de Senghor. II pèche par son caractère contradictoire. Le poète-philosophe écrit: "L'énergie étant force, il en résulte que c'est la radiale qui est créatrice, "l'étoffe première des choses", la tangentielle n'en étant qu'un produit résiduel» (SENGHOR, 1977, p. 72). Senghor reproche à Marx et à Engels d'avoir maintenu le «vieux dualisme des philosophes» en «donnant à la matière l'antériorité sur l'esprit». Or, en changeant les termes de l'antériorité (l'esprit précède et engendre la matière), il nous ramène à un système dualiste. Le monisme qu'il proclame n'en est donc pas un. Ce n'est qu'un «crypto-dualisme», pour reprendre l'expression de Nkrumah.

A notre avis, les cosmo-théosophies africaines postulent une vision métaphysique qu'on pourrait qualifier non de «naturalisme significationnel», mais plutôt de «réalisme significationnel». Cette doctrine affirme qu'il y a de l'Etre (réalisme) et que l'Etre porte en soi une «signification suprême» qui transcende la matière et l'esprit (significationnisme). Nous empruntons à Nsame Mbongo ce concept de «significationnisme», mais nous lui donnons un sens quelque peu différent. Le philosophe camerounais entend par cette notion une sorte de matérialisme ou de naturalisme dialectique immanent: «Cette capacité dialectique de la matière à se muer en son contraire, l'immatériel, à partir de la tension des forces qu'elle porte en elle, est sans doute sa signification la plus fondamentale et la plus générale. [...] La nature produit elle-même spontanément de la signification en son sein» (MBONGO, 2013, p. 132). Pour nous, au contraire, la signification profonde de l'Etre, bien qu'elle réside dans l'Etre, transcende toutes les déterminations ou manifestations de l'Etre (matière et esprit). La signification suprême de l'Etre, c'est l'Absolu. 


\section{CONCLUSION}

Les cosmo-théosophies examinées révèlent la complexité et la richesse de la pensée négro-africaine, surtout dans sa dimension métaphysique. La philosophie africaine contemporaine gagnerait à en faire un objet d'étude privilégié. Le seul risque à éviter, c'est celui des travers de l'ethnophilosophie. Les cosmo-théosophies ne sont pas le résidu d'une pensée collective inconsciente de soi, mais des tentatives d'explication rationnelle et symbolique du réel, élaborées par des Ecoles Initiatiques qu'il faut désormais considérer comme de véritables Cénacles du Savoir.

\section{RÉFÉRENCES}

BÂ HAMPÂTÉ, Amadou; DIETERLEN, Germaine. Koumen. Texte initiatique des Pasteurs Peul. Paris: EHESS, 2009.

BA MOCTAR, Cheikh. Etude comparative entre les cosmogonies grecques et africaines. Paris: L'Harmattan, 2007.

BIYOGO, Grégoire. Le mvett et la découverte de connaissances scientifiques nouvelles. In: MINKO BENGONE, Laurent. Comprendre autrement le Mvett. Paris: L’Harmattan, 2008.

CANEVA, Claudia; SINSIN, Mahougnon; THURUTHIYIL, Scaria. Filosofie in dialogo. Lexikon universale: India, Africa, Europa. Milano: Mimesis, 2017.

DIETERLEN, Germaine. Tendances de l'ethnologie française II. Paris: PUF, 1959.

DIOP, Cheikh Anta. Civilisation ou barbarie. Paris: Présence Africaine, 1981.

EFFA, Gaston-Paul. Le dieu perdu dans l'herbe. L'animisme, une philosophie africaine. Paris: Presses du Châtelet, 2015.

ELLOUÉ-ENGOUNE, Alain. Du Sphinx au Mvett. Connaissance et sagesse de I'Afrique. Paris: L'Harmattan, 2008.

FOURCHE, Tiarko; MORLIGHEM, Henri. Une bible noire: cosmogonie bantu. Paris: Les Deux Océans, 2002. 
GRIAULE, Marcel. Le savoir des dogons. Journal de la Société des Africanistes, n. XXIII, 1952.

GRIAULE, Marcel. Dieu d'eau. Entretiens avec Ogotemmêli. Paris: Fayard, 1966.

GRIAULE, Marcel; DIETERLEN, Germaine. Le renard pâle. Paris: Anté-Matière, 1991.

KAGAME, Alexis. La Philosophie Bantu comparée. Paris: Présence Africaine, 1976.

LALANDE, Alain. Vocabulaire technique et critique de la philosophie. Paris: Quadrige/Puf, 1996.

MBONGO, Nsame. La personnalité philosophique du monde noir. Contre-histoire de la philosophie. Paris: L'Harmattan, 2013. [Tome 2].

MENYOMO, Ernest. Les bases métaphysiques de la pensée négro-africaine. Paris: L’Harmattan, 2010.

NDOUTOUME, Tsira Ndong. Le Mvett. L'homme, la mort et l'immortalité. Paris: L’Harmattan, 1993.

NKRUMAH, Kwame. Le consciencisme. Paris: Présence Africaine, 1976.

OBENGA, Théophile. La philosophie africaine de la période pharaonique. 2780-330 avant notre ère. Paris: L’Harmattan, 1990.

OBENGA, Théophile. L'Egypte, la Grèce et l'Ecole d'Alexandrie. Histoire interculturelle dans l'antiquité. Aux sources égyptiennes de la philosophie grecque. Paris: L’Harmattan, 2005.

SENGHOR, Léopold Sédar. Liberté 3 - negritude et civilisation de l'universel. Paris: Le Seuil, 1977.

TOWA, Marcien. L'Idée d'une philosophie négro-africaine. Yaoundé: Clé, 1979. 